\title{
Integration of optical and satellite communication technologies to improve the cache filling time in future $5 \mathrm{G}$ edge networks
}

\author{
Alexis A. Dowhuszko ${ }^{\star}$, Senior Member, IEEE, Musbah Shaat ${ }^{\star}$, Senior Member, IEEE, \\ and Ana I. Pérez-Neira ${ }^{\star \dagger}$, Senior Member, IEEE \\ ${ }^{\star}$ Centre Tecnològic de Telecomunicacions de Catalunya (CTTC/CERCA), Barcelona, Spain \\ $\dagger$ Dept. Signal Theory and Commun., Universitat Politècnica de Catalunya (UPC), Barcelona, Spain \\ Tel: (+34) 93645 2900,Email: \{alexis.dowhuszko, musbah.shaat, ana.perez\}@cttc.es
}

\begin{abstract}
Caching is an enabler to avoid congestion and delay in the backhaul links of future $5 \mathrm{G}$ networks. The basic idea consists in estimating the content that would be most likely requested by the end users served by each edge node and, after that, make a pro-active placement of those files during the off-peak hours. Optical wired/wireless terrestrial links support a high point-to-point data rate but, when the same content needs to reach different destinations, parallel unicast transmissions are needed. On the other hand, satellite systems provide a lower link-level data rate but can easily implement a multicast transmission due to their wide-area coverage. In this paper, a resource allocation strategy that determines the most convenient way to transport the different content using both terrestrial (optical) and satellite (radio) technologies is proposed. Simulation results show that the placement time can be notably reduced in a hybrid terrestrial-satellite backhaul network, particularly in case of bad weather that impacts the data rate of wireless optical links. The effect of the file popularity distribution and the number of $5 \mathrm{G}$ edge nodes is also studied in detail.
\end{abstract}

Keywords: Free space optical; satellite systems, content delivery, caching, Zipf distribution, hybrid backhaul.

\section{INTRODUCTION}

The demand of mobile data that is expected in future $5 \mathrm{G}$ networks will be much higher than the one observed so far in $4 \mathrm{G}$. This imposes new challenges to the wireless access part of the $5 \mathrm{G}$ network, as well as to the backhaul links that connects the edge nodes to the core network. In order to mitigate the impact that the high data traffic of new services will have on the backhaul links, one possibility is to cache the most popular content at the $5 \mathrm{G}$ edge nodes which, apart from processing capabilities, will be also equipped with storage resources [1], [2]. This will bring the content closer to the end users, avoiding the transmission of popular content in peak hours and reducing the delivery time. However, the effective implementation of caching requires a dense mesh network of inter-connected edge nodes, to make an massive distribution of content in a timely manner.

In cases where optical fibers are already deployed [3], it is reasonable to re-utilize them to provide the backhaul connectivity of future $5 \mathrm{G}$ edge networks. However, due to cost of deploying new fiber cables may become prohibitive, particularly in dense urban areas or isolated sub-urban areas, Free Space Optical (FSO) technology appears as a convenient solution when a high-capacity link is needed to connect two edge nodes that are relatively close [4]. However, regardless the optical link is wired (fiber) or wireless (FSO), they are both more suitable for implementing a physical layer unicast transmission, which may create a serious problem in the total traffic load of the backhaul network when the same content needs to reach different destinations.

In this situation, the broadcast nature of satellite systems starts to gain importance, as they can reach many geographical distant destinations simultaneously with a minor adaptation of the physical layer parameters. This way, the same content that terrestrial optical (fiber/FSO) systems would have to unicast to multiple destinations can be easily offloaded to a single multicast satellite transmission. The delivery of content via satellite for $5 \mathrm{G}$ edge-catching has been investigated in the literature recently. For example, an intelligent caching algorithm compatible with multicast transmissions was proposed in [5], where the point-to-multipoint nature of the satellite system was exploited to place the different requested files based on their global and local popularity. Similarly, the authors of [6] proposed a mono-/multi-beam hybrid satellite-terrestrial architecture with caching to reduce the required time for content placement. Finally, a two-layer caching model, where caches in the ground stations constitute the first caching layer while the satellite cache forms the second layer, was proposed in [7].

This paper joins the current $5 \mathrm{G}$ research trend that seeks the integration of satellite and terrestrial technologies into a hybrid backhaul network, taking advantage of the complementary benefits that they have [8], [9]. However, in contrast to most of the work reported in the literature so far, this paper applies an adequate management of communication resources of both optical (terrestrial) and radio (satellite) communication systems to minimize the delivery time of the content to be cached. For this purpose, either a single multicast satellite or multiple unicast optical transmission is selected for each file to be placed according to its global popularity index. This way, the overall efficiency of the hybrid backhaul network is notably improved, with the possibility to adapt the 
delivery strategy according the 5G network parameters (e.g., number of edge nodes), Zipf-popularity distribution of the content, and instantaneous physical layer capacity of the optical and satellite links.

The rest of the paper is organized as follows: Section 2 presents the system model, the stochastic approach to determine the indexes of files to be placed in each $5 \mathrm{G}$ edge node (accoding to a given global popularity distribution), and the effect that weather conditions have on the FSO link data rates. Section 3 shows the simulation results, with emphasis on the improvement of the content delivery time when the use of both terrestrial (FSO) and satellite (radio) technologies is jointly optimized. Finally, conclusions are drawn in Section 4.

\section{SYSTEM MODEL}

The simplified system model of a $5 \mathrm{G}$ network that combines optical (mesh) and satellite (broadcast) links in the backhaul network is illustrated in Fig. 1. The system contains $K$ edge Base Stations (BS) whose associated users would (likely) request the delivery of $M$ files during the forthcoming peak-hour period, selected from a library containing $N$ elements. In addition to (at least) a pair of terrestrial FSO transceivers that connect the edge BS with the nearest neighbors, and through them to the core network in a multi-hop fashion, each BS is also equipped with a satellite dish to receive the content that should be proactively placed in its cache memory. In this paper, we assume that a pre-defined off-line caching algorithm has defined the indexes of files to be placed in each edge node [6], and that the core network has access to this information to determine the most convenient way to deliver the requested content using either a satellite multicast or FSO unicast transmission.

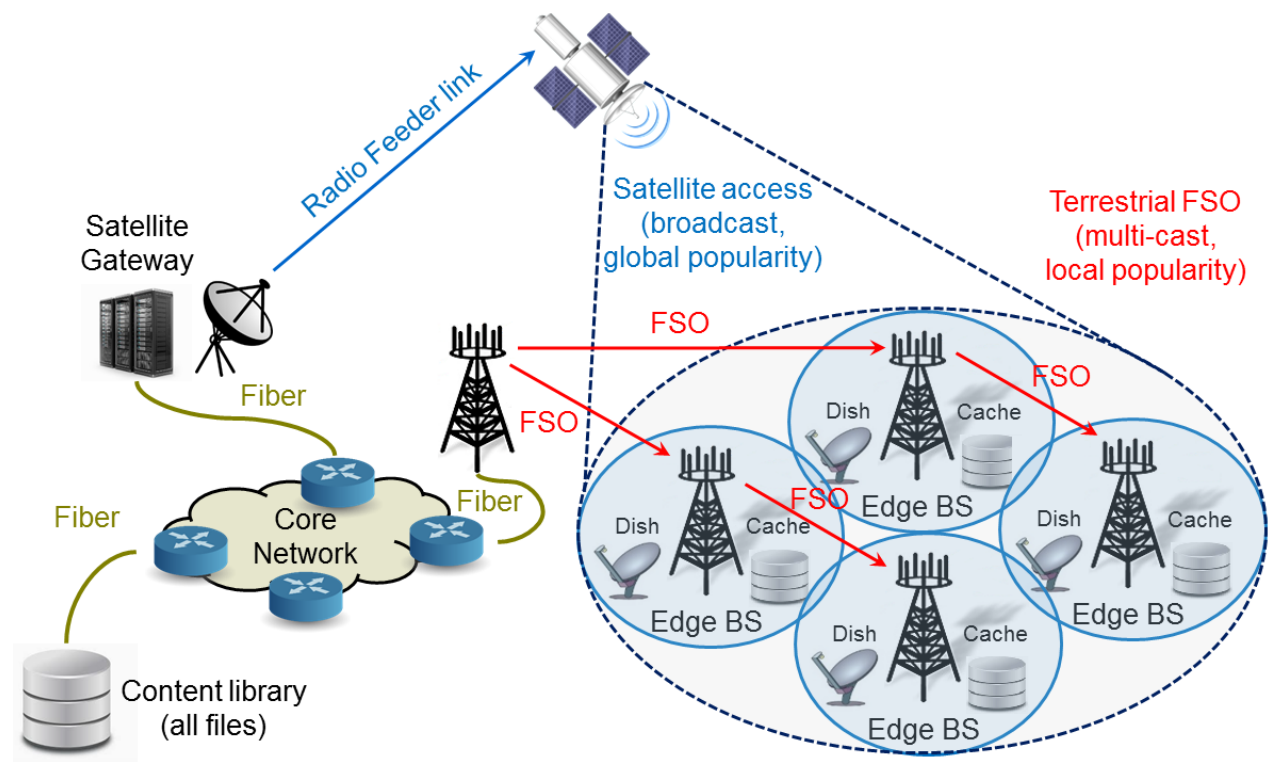

Figure 1: Hybrid terrestrial (FSO) and satellite (RF) backhaul network to deliver content into the $5 G$ edge.

\subsection{Popularity distribution of content}

It is well referenced in the literature that the popularity of requested files in a content delivery network follows a heavy-tail distribution, whose popularity trend can be accurately qualified by the Zipf's law [6] as

$$
p(n)=\frac{\Omega}{n^{\alpha}} \quad \Omega=\left(\sum_{n=1}^{N} \frac{1}{n^{\alpha}}\right)^{-1} .
$$

The parameter $\alpha$ controls the tail of the Zipf-distribution, such a low $\alpha$ implies the popularity distribution of files tends to be more homogeneous, whereas high $\alpha$ means that few files have very high popularity and a large number of files have very low popularity. Typical values of $\alpha$ are between 0.4 and 1.0. If we had $N=10000$ files, this would mean that $50 \%$ of requests occur for the top $25 \%$ and $6 \%$ of the content, respectively.

To generate the indexes of the files that each each BS $k$ in set $\mathcal{K}=\{1, \ldots, K\}$ requests in a given content delivery period, assuming that the global popularity of files is given by (1), the following algorithm is proposed:

1) For each file with index $n$ in set $\mathcal{N}=\{1, \ldots, N\}$, generate $G_{n}=p(n) \cdot M \cdot K$ Poisson points in the interval $[0, K]$ and tag each of this points with its corresponding file index. 
2) Determine the number of times that each file with index $n$ is requested locally at each edge BS $k$ (i.e., $\left.L_{n, k}\right)$, by summing the number of Poisson points with tag $n$ that lie in the interval $[(k-1), k)$.

3) Find the file indexes requested by each edge BS $k$ in a sample placement period, which will be contained in set $\mathcal{I}_{k}$. That is, $n \in \mathcal{I}_{k}$ if $L_{n, k} \geq 1$, whereas $n \notin \mathcal{I}_{k}$ otherwise.

4) Finally, the number of edge BSs requesting each file with index $n$ in a sample placement period (i.e., $B_{n}$ ) equals the number of sets $\mathcal{I}_{k}$ that contains the file index element $n$.

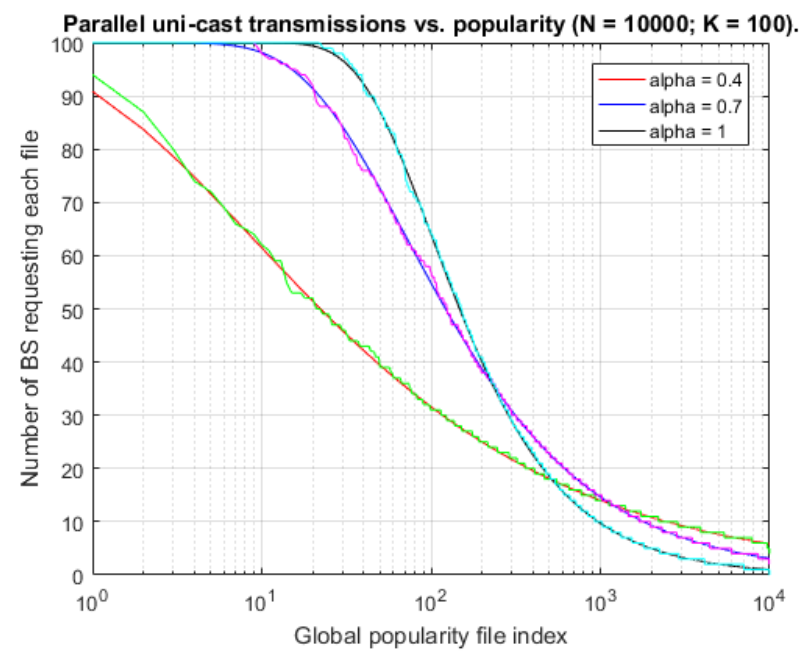

Figure 2: Number of times that each file should be downloaded for different popularity distributions.

Point values in Fig. 2 show the number of edge BSs that request file with index $n$ in a given placement period (i.e., snapshot of the stochastic experiment). Results are presented for different Zipf-parameters $\alpha$, to see the effect of different popularity conditions have on the number of times that each file needs to be transmitted over the backhaul. The solid lines that are also represented in the same figure were obtained using the following formula that has been derived using the theory of randomly distributed Poisson points in a given interval, i.e.,

$$
B(n)=\sum_{k=0}^{K} k \cdot P(k), \quad P(k)=\left(\begin{array}{c}
K \\
k
\end{array}\right) \exp (-\lambda(K-k))(1-\exp (-\lambda))^{k}, \quad \lambda=p(n) \cdot M,
$$

where $p(n)$ is given in (1).

\subsection{Data rate in free space optical links}

Without loss of generality, we assume that the end-to-end data rate of the multi-hop FSO terrestrial link that is configured to connect each edge BS with the core network is limited by the data rate of the longest link. More accurate model includes studying the routing and flow control of the overall network as provided in our work in [10] is skipped due to space limitation.

Given a laser transmitted power $P_{\mathrm{tx}}=18 \mathrm{dBm}$ at optical wavelength $\lambda_{\mathrm{fso}}=1550 \mathrm{~nm}$ used to cover a wireless link distance of $d_{\mathrm{fso}}$. Let us assume that the aperture of the receive telescope is $A_{\mathrm{rx}}=13 \mathrm{~mm}$ and that the beam divergence of the transmitter is $\theta_{\mathrm{tx}}=8.5 \mathrm{mrad}$. Then, is the receive sensitivity of the FSO link for $1.25 \mathrm{Gbps}$ was designed to be $N_{\mathrm{b}}=-36 \mathrm{dBm}$ (or equivalently, 1568 photons/bit), the achievable data rate of the FSO link can be obtained as

$$
R=\frac{P_{\mathrm{tx}} \tau_{\mathrm{opt}} \tau_{\mathrm{atm}} A_{\mathrm{rx}}}{\pi\left(\theta_{\mathrm{tx}} / 2\right)^{2} d^{2} E_{\mathrm{p}} N_{\mathrm{b}}},
$$

where $E_{\mathrm{p}}=h c / \lambda_{\mathrm{fso}}$ is the photon energy. Therefore, for different weather conditions, such as clear sky $(\alpha=0.2 \mathrm{~dB} / \mathrm{km})$, haze $(\alpha=4 \mathrm{~dB} / \mathrm{km})$, and fog $(\alpha=21 \mathrm{~dB} / \mathrm{km})$, the achievable data rate of the FSO link for different link distances are notably affected.

\section{PERFORMANCE EVALUATION}

Figure 3 show the file placement time that is required when either the FSO terrestrial network (solid lines) or satellite network (dashed lines) are used to place the files requested by the different edge BSs for different Zipf distributions. As expected, the lower is the value of $\alpha$, the longer is the delivery time as a larger number 
of files has to be stored in the edge BSs (the area below the curves in Fig. 2 would be bigger for lower $\alpha$ if the $\mathrm{x}$-axis were represented in linear scale). In addition, it is possible to observe that the atmospheric visibility affects notably the delivery time of FSO links, e.g., when comparing the performance in presence of clear sky (left panel) and haze (right panel). In contrast, the file delivery time using the satellite system is always the same, as the multicast transmission strategy is transparent to the popularity distribution parameter $\alpha$ and the number of BSs in the 5G edge network (we have also assumed that the satellite data rate is not affected by weather conditions, regardless there is rain, haze or fog). Finally, the points in which the solid and dashed lines cross represent the optimal strategy to minimize the delivery time of the requested content using both multi-cast satellite transmission (for most popular content) and FSO terrestrial transmissions (for least popular content).

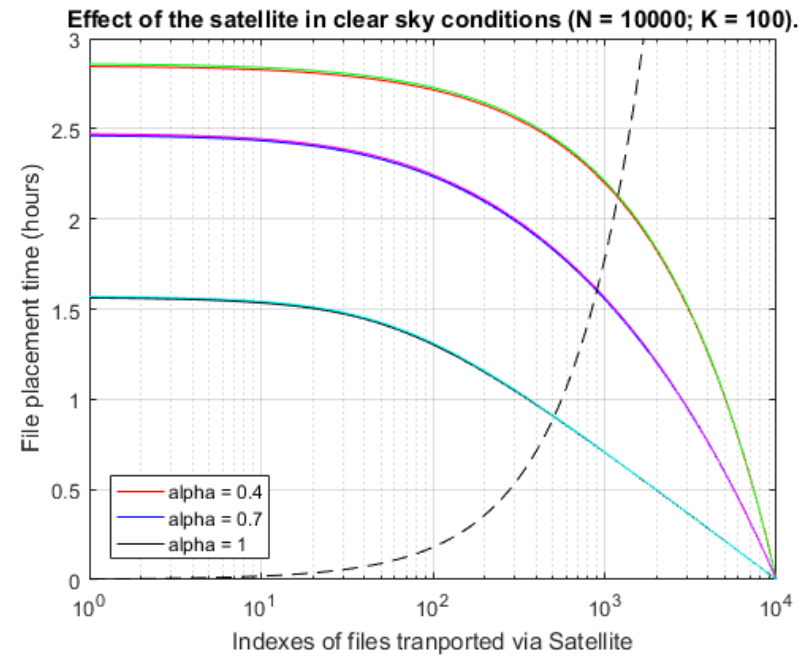

(a) Clear sky conditions (visibility: $23 \mathrm{~km}$ )

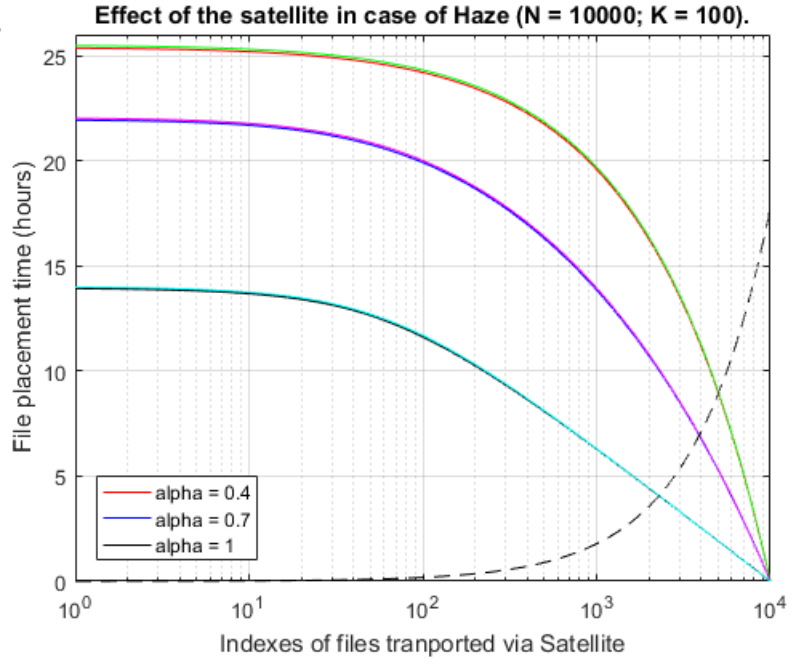

(b) Haze (visibility: $2 \mathrm{~km}$ )

Figure 3: File placement time of the different backhaul technologies assuming different weather conditions. Solid lines: FSO (terrestrial). Dashed line: Radio (Satellite)

Finally, Fig. 4 show the minimum delivery time that is required when both FSO terrestrial (unicast) and satellite (multicast) systems are utilized jointly to minimize the delivery time of the content requested by the edge BSs. In this figure, simulation results are presented for different numbers of edge BSs (served by the same satellite spot beam), and for different global popularity distributions of the files. As expected, the placement time reduction becomes more notable as the number of edge BSs grows, and as the popularity distribution indexes $\alpha$ reduces. For example, when $K=500$ edge BSs request the placement of files whose popularity is determined by a Zipf-distribution with a ligth tail (i.e., $\alpha=1$ ), the placement time is reduced in a factor of 2.5 when using the FSO and Satellite systems for backhauling (i.e., from 8 hours to about 3 hours). The opposite situation takes place when $K=50$ and $\alpha=0.4$, as the gain that is observed is in the order of $20-25 \%$.

\section{CONCLUSIONS}

In this paper we proposed a resource allocation strategy to minimize the delivery time of the content requested for off-line caching at the edge nodes of a $5 \mathrm{G}$ network. We considered that the indexes of the files that are requested by each edge node are known before the placement period starts, and that the popularity distribution of each file can be approximated accurately using a Zipf-distribution with a given parameter $\alpha$. Then, combining this information with other relevant configuration parameters of the backhaul network, such as the number of edge BSs and the end-to-end data rate of the multi-hop FSO links, it was possible to determine the fileindex-threshold that defined the disjoint sets of files to be multicast transmitted over the satellite system and unicast transmitted over the FSO terrestrial system, respectively. Based on the obtained simulations results, it was possible to conclude that the broadcast nature of satellite communication systems enables to reduce the file placement time notably in an hybrid backhaul network, particularly when the number of edge BSs is large, the popularity is concentrated in few files, and the weather conditions are not favorable for FSO propagation.

\section{ACKNOWLEDGMENTS}

This work has been funded by the Ministry of Science, Innovation, and Universities of Spain under Project TERESA-TEC2017-90093-C3-1-R (AEI/FEDER, UE), and by the Catalan government under grant 2017 SGR 1479. 


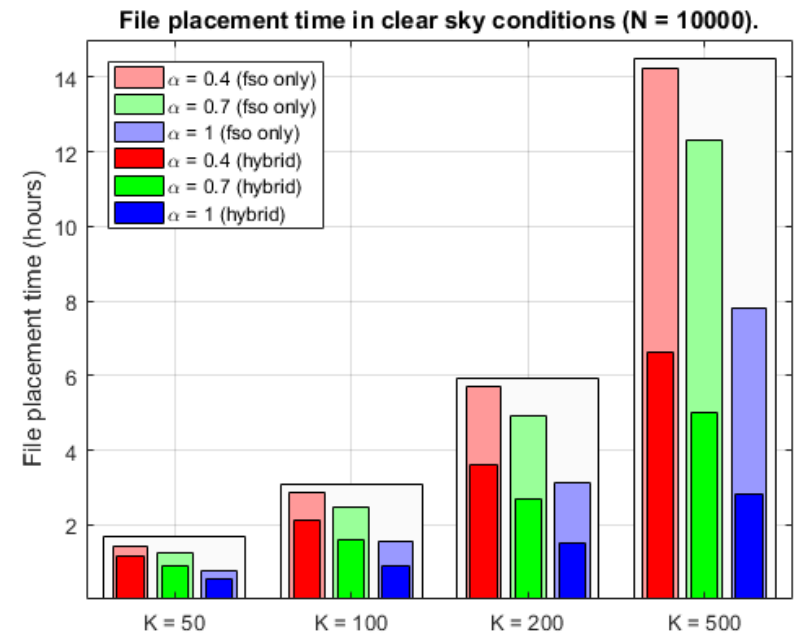

Figure 4: Time delivery time for different popularity distributions and number of base stations.

\section{REFERENCES}

[1] E. Bastug, M. Bennis, and M. Debbah, "Living on the edge: The role of proactive caching in $5 \mathrm{~g}$ wireless networks," IEEE Commun. Mag., vol. 52, no. 8, pp. 82-89, Aug. 2014.

[2] W. Han, A. Liu, and V. Lau, "Phy-caching in 5G wireless networks: Design and analysis," IEEE Commun. Mag., vol. 54, no. 8, pp. 30-36, Aug. 2016.

[3] A. Dowhuszko, J. Fábrega, and A. Pérez-Neira, "Integration of wireless and optical technologies to meet the requirements of 5G networks and beyond," in Proc. 39th Meeting of the Wireless World Research Forum, Oct. 2017, pp. 1-8.

[4] I. Son and S. Mao, "A survey of free space optical networks," Digital Communications and Networks, vol. 3, no. 2, pp. 67-77, May 2017.

[5] C. Brinton, E. Aryafar, S. Corda, S. Russo, R. Reinoso, and M. Chiang, "An intelligent satellite multicast and caching overlay for CDNs to improve performance in video applications," in Proc. AIAA Int. Commun. Satellite Systems Conf., Oct. 2013, pp. 1-9.

[6] A. Kalantari, M. Fittipaldi, S. Chatzinotas, T. Vu, and B. Ottersten, "Cache-assisted hybrid satelliteterrestrial backhauling for 5G cellular networks," in Proc. IEEE Global Commun. Conf., Dec. 2017, pp. $1-6$.

[7] H. Wu, J. Li, H. Lu, and P. Hong, "A two-layer caching model for content delivery services in satelliteterrestrial networks," in Proc. IEEE Gobal Commun. Conf., Dec. 2016, pp. 1-6.

[8] M. Shaat, E. Lagunas, A. Pérez-Neira, and S. Chatzinotas, "Integrated terrestrial-satellite wireless backhauling: Resource management and benefits for 5G," IEEE Veh. Tech. Mag., vol. 13, no. 3, pp. 39-47, Sept. 2018.

[9] R. Gopal and N. BenAmmar, "Framework for unifying 5G and next generation satellite communications," IEEE Network, vol. 32, no. 5, pp. 16-24, Sept. 2018.

[10] M. Shaat and A. Pérez-Neira, "Joint flow control and link scheduling in hybrid terrestrial-satellite wireless backhauling network," in Proc. IEEE Int. Conf. Commun. Workshops, May 2017, pp. 870-875. 